\title{
Numerical and experimental study of the effect of microslits on the normal absorption of structural metamaterials
}

\author{
H. Ruiz ${ }^{1}$, C.C. Claeys ${ }^{1}$, E. Deckers ${ }^{1}$, W. Desmet ${ }^{1}$ \\ ${ }^{1}$ KU Leuven, Department Mechanical Engineering \\ Celestijnenlaan 300 B, B-3001, Heverlee, Belgium \\ e-mail: heidi.ruiz@kuleuven.be
}

\begin{abstract}
Resonant metamaterials are emerging as novel concepts to reduce noise levels in targeted frequency zones, so-called stop bands. The metamaterial concept improves acoustic behaviour through an increase of the insertion loss. This paper concerns a first investigation on the absorption capabilities of a resonant metamaterial when thermo-viscous effects are incorporated via the addition of microslits. In a previous work, a resonant metamaterial was obtained through the inclusion of resonating structures into cavities of an open honeycomb assembly. In this study, the air gap of the honeycomb structure is reduced so as to provide viscous losses for the travelling waves. Considering that the created resonant structures with open cavities are rigid, an equivalent fluid model is used to calculate the acoustical properties of a so called microslit metamaterial. It is demonstrated that the unit cell structure can be divided into parallel elements for which the acoustic impedance can be computed via the transfer matrix approach TMM in parallel and series. Likewise, it is shown that the structural response can be predicted by FEM models allowing studying the structural effects separately from the viscous-thermal effects predicted by the equivalent fluid model. Moreover, the combined effect of both approaches is shown experimentally where it is observed that: (i) the absorption of the resonant metamaterial is increased by the addition of microslits, (ii) the modes of the test sample appear as small peaks on the absorption curve of the microslit metamaterial, (iii) the structural modes are grouped below and above the stop band and, (iv) the resonant structures do not lead to additional absorption in the stop band region. Analytical models are compared to experimental measurements to validate the models and to show the potential of this material assembly.
\end{abstract}

\section{Introduction}

In response to increasing customer expectations and more restrictive regulations, the acoustical behaviour of consumer goods and machinery is gaining ever more attention. Classical heavy and/or bulky solutions to improve acoustic behaviour, such as heavy layers or absorptive foams, are often undesirable given the trend towards compact and lightweight design, triggered by ecological and economic reasons. In search for novel material systems that combine lightweight characteristics with good acoustical behaviour, resonant metamaterials come to the fore, be it at least in some targeted and tuneable frequency ranges, referred to as stop bands. Resonant metamaterials with stop band behaviour are obtained through the inclusion of resonant cells on a scale smaller than the structural wavelengths to be influenced, opening up the path towards compact low frequency vibro-acoustic solutions [1]. Reference works such as [2,3], show that the working principles of metamaterials can be described based on the Fano-type interference between incoming waves and the waves re-radiated by the resonant cells. Their acoustic 
properties have been studied by Liu et al. who showed that local resonant metamaterials lead to an increased acoustic transmission loss through reflection and thus are not based on the improvement of acoustic absorption [4]. However, the possibility of acoustic absorbing metamaterials has been discussed as well by Mei et. al. who achieved absorption through large local structural deformation in the resonant cells combined with a dissipative material in the zone of deformation [5]. This was accomplished through the combination of membrane inclusions with steel inserts.

High absorption coefficients can be achieved in structures that can provide large values of acoustic resistance at the same time as low acoustic mass reactance [6]. A common solution to generate acoustic absorption is by means of poroelastic materials, like foams [7]. These are effective for airborne sound mitigation through dissipation of the acoustic waves into heat. Their working frequencies are often dependent on the thickness of the material; to be effective the thickness has to equal at least a quarter of the acoustical wavelength to be influenced, restricting their use to the mid and high frequency range. To improve the absorption performance, a mass resistance and reactance can be added to the impedance of the absorber by placing a perforated panel at the upstream side [8]. The effect is not only a shift of the frequencies of maximum absorption to a lower frequency range but an increase of the absorption peak as well. By reducing the diameter of the perforation to the sub millimeter size, the panel itself can provide energy dissipation of the sound waves into heat by viscous and thermal losses inside the perforations as a microperforated panel (MPP) [9].

In this paper a metamaterial with improved acoustic absorption behaviour is pursued. As starting point an already validated metamaterial design is used. In this design, existing air gaps are redesigned to micro slits in order to increase visco-thermal losses, similar as in slitted panels [10]. The resonant structures of the metamaterial are unchanged and apart from a possible increase in damping of the cell resonances due to the microslits, the stop band behaviour should be unchanged. In this way, a microslit metamaterial is obtained.

For the design and numerical prediction of this microslit metamaterial, different numerical techniques are applied. To assess the stop band behaviour, it is assumed that the metamaterial is periodic; however, this is not required from a physical point of view. From literature it is known that, based on an undamped Finite Element (FE) model of the unit cell of a periodic structure and the application of periodicity boundary conditions, dispersion curves for freely propagating structural waves in an infinite periodic structure can be derived [11,12]. These dispersion curves allow identification of the stop band region which corresponds to frequency zones for which no free wave propagation solutions are found.

The calculation of the acoustic absorption provided by the material is done using the JohnsonChampoux-Allard (JCA) model $[13,14]$ which allows handling the structure-air combination in the microslit metamaterial as an equivalent fluid. The absorption peak of the microslit metamaterial is tuned by the combined effects of the diameter of the slits, the ratio of open area to total area, the thickness of the layer and a backing cavity filled with air when the metamaterial is situated in front of a rigid wall. The acoustic impedance of a resonant structure with microslits inlaid in a honeycomb structure backed by a cavity filled with air is predicted with the transfer matrix method (TMM) in series and parallel configurations [15].

The paper is outlined as follows. The design of the metamaterial is described in Section 2. Section 3 discusses the equivalent fluid modelling and the layered TMM representation. Section 4 elaborates on the results and Section 5 presents the conclusions. 


\section{Increasing visco-thermal losses of a local resonant metamaterial}

To obtain a material that combines stop band behaviour with acoustic absorption, the geometry of an already validated metamaterial is used as a starting point [Error! Reference source not found.]. This geometry and the rationale behind the design are described first. Next, the modifications to this geometry are discussed in view of increasing the acoustic absorption and simplifying the models of this structure. The last section describes how the resonance frequency of the resonant structures is tuned with respect to the expected peak frequency of acoustic absorption.

\subsection{Geometry of the original metamaterial}

A metamaterial based on resonant cells as described in [Error! Refe rence source not found.] is taken as the basis structure of study (Figure 1). The metamaterial consists of a host structure with a rectangular core, to which resonant structures have been added to introduce stop band behaviour. Since the goal of this metamaterial is to increase sound transmission loss, a skin has been added on one side such that no acoustic waves can directly pass through the structure. Figure 2 shows different perspectives of the unit cell of this metamaterial: the total structure is built as a repetition of this unit cell in the $\mathrm{X}$ and $\mathrm{Y}$ directions. Table 1 describes the dimensions of the unit cell and the resonant structure.

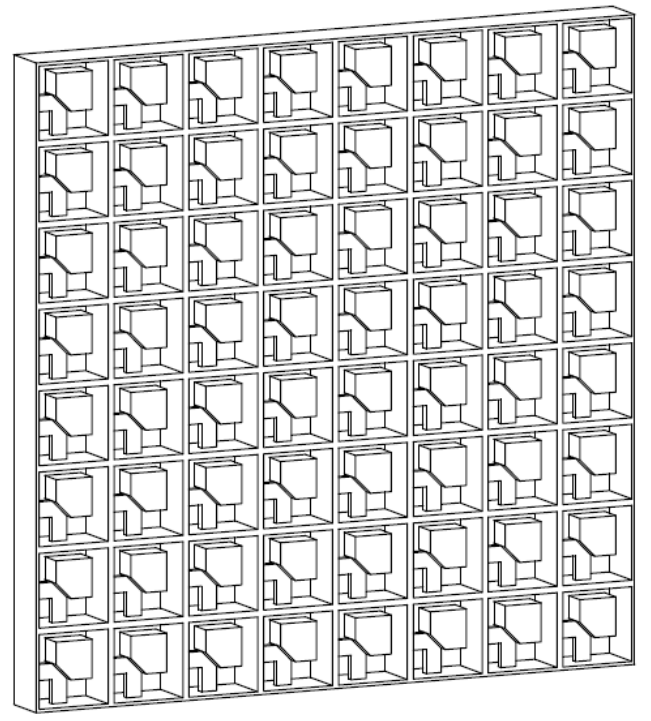

Figure 1: Example of a metamaterial panel based on inclusion of resonant structures.

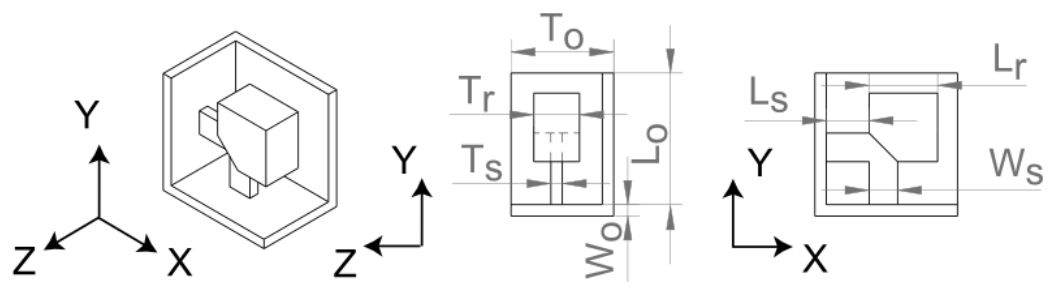


Figure 2: Different perspectives of the unit cell of the acoustic metamaterial according to [Error! Reference source not found.]. Table 1 lists the dimensions of this unit cell.

\begin{tabular}{l|l|c} 
Dimension & Value (mm) & Abbreviation \\
\hline Length of the inside of the unit cell & 11.5 & $L_{o}$ \\
Thickness of cell sides & 1 & $W_{O}$ \\
Depth of the inside of the unit cell & 9 & $T_{o}$ \\
Length of the resonant structure mass & 6 & $L_{r}$ \\
Thickness of the resonant structure mass & 4 & $T_{r}$ \\
Length of the connection legs & 3.75 & $L_{S}$ \\
Width of the connection legs & 2.5 & $W_{S}$ \\
Thickness of connection legs & 1 & $T_{S}$
\end{tabular}

Table 1: Geometry of the unit cell of the original metamaterial as shown in Figure 2.

The resonant structure is designed to have a pronounced low frequent bending mode. To achieve this, a design similarly to a spring mass-system is used; a thicker part i.e., the mass, is connected to the core of the host structure through two connection legs i.e. the springs. To increase the resonance frequency of the resonant structure either the leg thickness can be increased or the mass thickness can be decreased. Figure 3 shows the deformation of the first bending modes of the resonant structure computed with an FE software [Error! Refe rence source not found.]. The combination of this resonant structure with the host structure, as shown in Figure 1, leads to stop band behaviour in the vicinity of the resonance frequency of the resonant structure and provides the possibility to combine high acoustic transmission loss in the low to mid frequency region together with excellent mechanical properties.

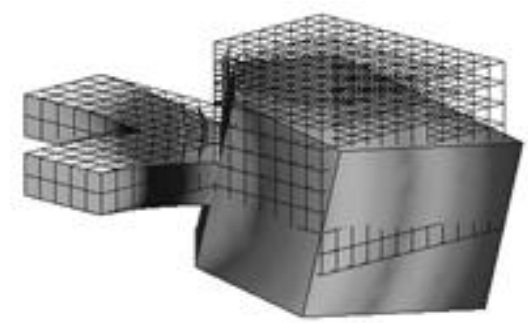

Figure 3: Visualisation of the bending mode of the resonant structure according to Figure 2. The undeformed mesh is shown against a shaded deformed mesh.

The metamaterial concept has a rather complex geometry and topology which is hard to produce with traditional manufacturing processes. Additive manufacturing is chosen as production process since this allows fabricating complex parts without the need of an expensive mould, making it a suitable production process for prototype design. The test samples discussed in this paper are produced by the use of selective laser sintering of Polyamide. The material parameters according to the manufacturer are listed in Table 2. The production of the test samples is further discussed in Section 4.

\begin{tabular}{l|l} 
Parameter & Value \\
\hline Elastic modulus & $1750 \mathrm{MPa}$
\end{tabular}


\begin{tabular}{l|l} 
Poisson ratio & 0.3 \\
Density & $950 \mathrm{~kg} / \mathrm{m}^{3}$
\end{tabular}

Table 2: Material parameters of the polyamide used for production of the test samples.

\subsection{Adapting the unit cell to increase visco-thermal losses}

The air gap between the resonant structure and the host structure in the original design does not has any effect on the resonance frequency of the resonant structure nor contributes to the transmission loss of the metamaterial. However, with an appropriate use of this space, the acoustic absorption properties of the metamaterial might be enhanced. One way of doing so is modifying the geometry of the unit cell so that energy losses in airborne sound waves can happen. Besides fulfilling this condition and in order to ease the modeling, two additional changes are made to the design of the unit cell. First, the geometry is adapted such that a layered modelling approach is more straightforward without changing the resonant behaviour of the resonant structure. To do so, the connection legs are not attached in the middle of the resonant structure mass, but are flush mounted parallel to the bottom face of this mass (Figure 4: ). Secondly, the skin on the host structure is removed and is replaced by a rigid back plate on the test set-up, such that the distance between host and skin is substituted by an adjustable cavity filled with air. Finally, the height of the host structure of the unit cell $\left(T_{o}\right)$ is set equal to the thickness of the resonant structure mass $\left(T_{r}\right)$.

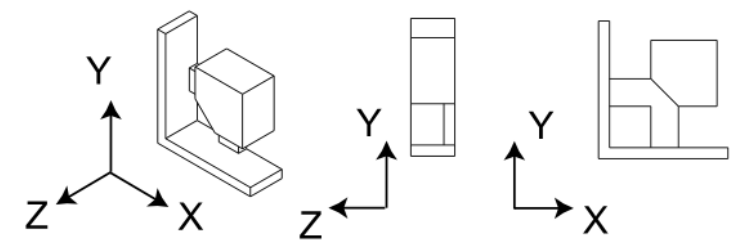

Figure 4: Adapted metamaterial geometry.

The structure shown in Figure 4: is a unit cell whose outer core is separated from the resonant body by irregular air sections. It is observed that the space between the resonant structure and the host structure is large compared to the typical sizes for microperforations and thus no acoustic dissipation due to visco-thermal effects is observed. In order to provide acoustic absorption by viscous dissipation, the lay-out of the host structure is changed such that the air gaps in the unit cell are decreased and only a slit of air with width $d$ between the resonant structure and the host structure remains.

Two types of geometry are proposed:

1. The first type is a constant thickness resonant structure lay-out, in which the height of the host structure $\left(T_{o}\right)$ and the thicknesses of both the mass $\left(T_{r}\right)$ and the connection legs $\left(T_{s}\right)$ are all the same.

2. The second type is a non-constant thickness resonant structure lay-out in which the height of the host structure $\left(T_{o}\right)$ is equal to the mass thickness $\left(T_{r}\right)$ but the connection legs $\left(T_{s}\right)$ are thinner. This allows more freedom to design the resonance frequency of the resonant structure.

The motivation for these two different types of geometry comes from a modelling point of view. As will be explained in the next section, the acoustic absorption of a non-constant thickness lay- 
out is less straightforward to model. Therefore the constant thickness lay-out is conceived first and the complexity is increased gradually.

The constant thickness lay-out is obtained by a resonant structure separated from an outer core by microslits as shown in Figure 5.

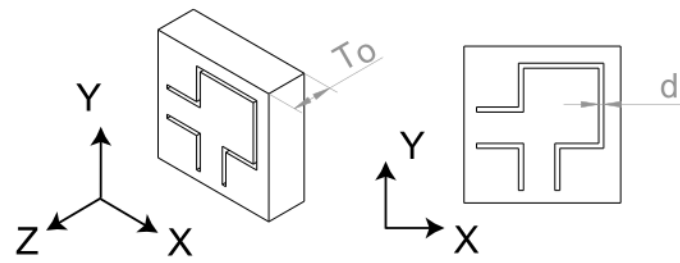

Figure 5: 3D and front view of a unit cell with a constant thickness resonant structure.

In the case of a non-constant thickness resonant structure the unit cell is somewhat different. It is chosen to change the depth of the added material to decrease the air gap between the resonant structure and the host structure dependent on the location. A diagonal line is traced inside the unit cell, separating the mass part from the legs. The material added between the original host structure and the resonant structure mass has the same thickness as the resonant structure mass. On the contrary, the material added to the part near the legs and between the legs and the diagonal division has the same thickness as the legs. Figure 6 shows a picture of such a unit cell.

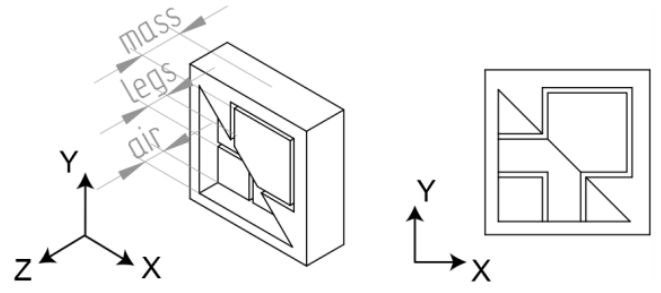

Figure 6: 3D and front view of a unit cell with a non-constant thickness resonant structure.

The constant thickness resonant structure can be seen as a microperforated slit panel and the same can be said for the non-constant thickness resonant structure when observing it from the back view. In this way, a wideband absorber can be achieved if the perforations are in the submillimetric range and the panel is placed in front of a rigid wall backed with a cavity filled with air [9].

\subsection{Choosing the resonance frequency of the resonant structure}

On the one hand, it is expected that the dimensions of the microslits in combination with the depth of the back cavity will lead to a peak frequency for acoustic absorption (see Section 3). On the other hand, the resonance frequency of the resonant structure will lead to a stop band. Both phenomena can be positioned relative to each other in the frequency domain. While the back cavity can be tuned in the test set-up so the absorption frequency caused by the microslits can be varied during the test with a certain amount, the resonance frequency of the resonant structure will be fixed by the design and can thus not be altered on the test set-up.

It is chosen to design for each lay-out, constant and non-constant thickness, two different resonant structures, such that a so called lower and a higher frequency stop band can be achieved. 
This allows investigating whether a beneficial effect can be reached if the stop band is placed below, at or above the peak absorption frequency, and to validate the numerical models.

As stated in Section 1, starting from an FE model of the unit cell, the stop band frequencies of the equivalent infinite metamaterial can be derived. By applying the Bloch-Floquet periodicity conditions to the boundaries of the unit cell, the infinite structure is accounted for. The solution of the associated eigenfrequency problem allows constructing dispersion curves for free wave propagation. Frequencies for which no solutions for dispersion curves are found correspond to zones without free wave propagation and consequently a stop band region. A MatLab routine is used to derive these dispersion curves based on the stiffness and mass matrices obtained from the FE model of the unit cell.

Given the dimensions of the legs, $2.5 \times 3.75 \times 4 / 2 / 1 \mathrm{~mm}$, solid elements are necessary to capture the behaviour of the resonant structure. Linear hexagonal (Lin. Hex.) elements are chosen for the solid elements. The maximal element length is $0.5 \mathrm{~mm}$, and always at least 3 elements are used across the thickness of the resonant structure legs. In the case of a constant thickness lay-out with a leg thickness of $1 \mathrm{~mm}$, linear shell (Lin. Shell) elements are used to speed up the calculations. Table 3 lists the constitution of the different models, the geometry of the resonant structure and the expected stop band frequencies.

\begin{tabular}{|c|c|c|c|c|}
\cline { 2 - 5 } \multicolumn{1}{c|}{} & \multicolumn{2}{c|}{ Constant thickness } & \multicolumn{2}{c|}{ Non-constant thickness } \\
\cline { 2 - 5 } \multicolumn{1}{c|}{} & Low freq. & High freq. & Low freq. & High freq. \\
\hline$T_{r}$ & $1 \mathrm{~mm}$ & $4 \mathrm{~mm}$ & $4 \mathrm{~mm}$ & $4 \mathrm{~mm}$ \\
$T_{S}$ & $1 \mathrm{~mm}$ & $4 \mathrm{~mm}$ & $1 \mathrm{~mm}$ & $2 \mathrm{~mm}$ \\
Nodes & 809 & 7281 & 6764 & 6397 \\
Elements & 691 Lin. shell & 5528 Lin. Hex. & 4851 Lin. Hex. & 4616 Lin. Hex. \\
Stop band & $1526-1968 \mathrm{~Hz}$ & $4589-5883 \mathrm{~Hz}$ & $1011-1180 \mathrm{~Hz}$ & $2374-2841 \mathrm{~Hz}$ \\
\hline
\end{tabular}

Table 3: Geometry and numerically predicted stop band frequencies of the different microslit metamaterial samples.

\section{Modelling of the microslit metamaterial}

This section discloses the analogy of the microslit metamaterial with a slitted panel. Under certain conditions, the acoustical impedance of the proposed material can be attained through the use of models for microperforated slit panels $[17,10]$. Nevertheless it is also possible to use an equivalent fluid model to account for the fluid-structure interaction inside the material as in [7, 18]. This last approach is taken to be more suitable for the cases studied and will be explained in the next section.

\subsection{Equivalent fluid modelling}

Local resonant metamaterials appear as a combination of a rigid resonant body embedded in a soft media as in [19]. In the same manner, the microslit metamaterial is established comprising an effective complex density $\rho_{\mathrm{e}}(\omega)$ and an effective complex bulk modulus $K_{\mathrm{e}}(\omega)$ that allow to characterize it acoustically depending on the media in which it is interacting with. The precise 
values of the temperature, pressure, density, viscosity and speed of sound in the media at the time of measurement are therefore fundamental to attain a good characterization. However, it is also essential to identify the geometrical parameters of the material which include: the porosity $\phi$, the geometric tortuosity $\alpha_{\infty}$, the flow resistivity $\sigma$ and the thermal $\Lambda^{\prime}$ and viscous $\Lambda$ characteristic lengths $[13,14]$. The evaluation of the unit cell allows determining each one of these parameters. The key values to start with are the shape and thickness of the pores.

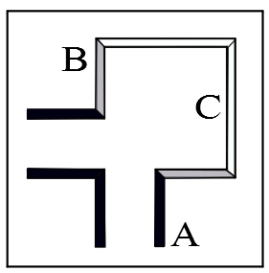

Figure 7: Schematic representation of the different parallelepiped slit-like shapes within the unit cell: A (black), B (gray) and C (white).

Figure 7 reveals three parallelepiped slit-like shapes around the resonant structure whose areas are: $A=d\left(L_{s}-d / 2\right), B=d\left(L_{r}-w_{s}\right)$ and $C=d\left(L_{r}+\mathrm{d}\right)$. In the case of the constant thickness lay-out, the total open area $O_{A}$, wetted perimeter $\mathrm{W}_{p}$ and hydraulic radius $R_{h}$ of the unit cell yield,

$$
\begin{gathered}
O_{A}=4 A+2 B+2 C, \\
\mathrm{~W}_{p}=20_{A} / d+4 d \text { and } \\
r_{h}=0_{A} / \mathrm{W}_{p} .
\end{gathered}
$$

For the non-constant thickness case the distribution of the open area inside the unit cell is done differently. The diagonal that separates the mass from the legs, divides the unit cell into two zones, the bottom part whose area is $T_{A l}$ and the upper zone whose area is $T_{A m}=T_{A}-T_{A l}$ with $T_{A}=L_{o}{ }^{2}$. The open area of the legs $O_{A l}$ and mass $O_{A m}$ are respectively $O_{A l}=4 \mathrm{~A}$ and $O_{A m}=$ $2 B+2 C$. Likewise, the wetted perimeter and hydraulic radius in each zone are calculated as $W_{p A l}=4 L_{s}+2 d, \quad r_{h A l}=4 L_{s} d / W_{p A l}, \quad W_{p A m}=4 L_{r}-2 w_{s}+2 d \quad$ and $\quad r_{h A m}=d\left(4 L_{r}-\right.$ $\left.2 w_{s}\right) / W_{p A l}$. This subtlety of separating the areas of the mass and the legs is needed for the accurate prediction of the absorption coefficient of equivalent fluids in parallel as will be described in Section 3.2.

The five geometric parameters together with the effective density and bulk modulus of each zone can be calculated with the appropriate use of the open areas, wetted perimeters and hydraulic radiuses. The first parameter that can be determined from the known areas is the porosity $\phi$ which is the ratio of the open area to the total area. In this case it is defined as $\phi_{c}$ for the constant thickness resonant structure and $\phi_{A l}, \phi_{A m}$ for the two zones of the non-constant thickness case

$$
\begin{gathered}
\phi_{c}=\frac{\mathrm{O}_{A}}{\mathrm{~T}_{A}}, \\
\phi_{A l}=\frac{\mathrm{O}_{A l}}{\mathrm{~T}_{A l}} \\
\phi_{A m}=\frac{\mathrm{O}_{A m}}{L_{o}^{2}-\mathrm{T}_{A l}} .
\end{gathered}
$$

The path of the flow through the structure can be described by the geometric tortuosity which is 1 plus a correction for the radiation of air at the sides of the structure. When the material is surrounded by air at the upstream and downstream sides, the geometric tortuosity is calculated as 


$$
\alpha_{\infty}=1+\frac{2 \varepsilon}{\mathrm{t}},
$$

where $t$ is the thickness of the layer and $\varepsilon$ is a correction length that is a function of the porosity and the open area [20]:

$$
\varepsilon=0.48 \sqrt{\mathrm{O}_{A}}(1-1.25 \sqrt{\phi}) .
$$

The length correction given by equation 4 is valid for $\sqrt{\phi}<0.4$. Due to the manufacturing process and the melting of the polyamide by the laser sintering, the edges of the slits present rounded shapes as seen in Figure 8.
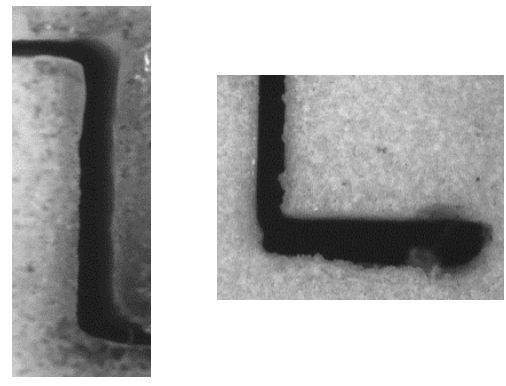

Figure 8: Microscopic views of slits surrounding the resonant structure mass and legs.

It is therefore expected that the correction length diminishes as compared to the sharp edge calculation of Eq. (4). Hence, for the equivalent fluid modelling, the open area with rounded edges is taken as $\pi r_{h}{ }^{2}$ and the correction length becomes $\varepsilon=0.48 \sqrt{\pi r_{h}^{2}}(1-1.25 \sqrt{\phi})$.

The next parameter that can be calculated from the porosity and known areas is the flow resistivity, which for a rectangular slit yields [7]

$$
\sigma=\frac{12 \mu}{r_{h}^{2} \phi}
$$

where $\mu$ is the dynamic air viscosity. The viscous characteristic length is calculated as

$$
\Lambda=\sqrt{\frac{8 \alpha_{\infty} \mu}{\phi \sigma}} \frac{1}{c_{\mathrm{r}}}
$$

where $c_{\mathrm{r}}$ is a constant defined for the pore shape which is 0.81 for slits. Yet, the rounded edges decrease the value of $\Lambda$ and $c_{\mathrm{r}}$ approaches to 1 as in a cylindrical pore with circular cross section. Finally, the thermal characteristic length is obtained by

$$
\Lambda^{\prime}=2 O_{A} / W_{p}
$$

Equations 2 to 7 allow the calculation of the equivalent density and bulk modulus as

$$
\begin{gathered}
\tilde{\rho}_{e}(\omega)=\rho_{0} \alpha_{\infty}\left(1+\frac{\sigma \phi}{j \omega \rho_{0} \alpha_{\infty}} \sqrt{\left(1+j \frac{4 \omega \rho_{0} \alpha_{\infty}^{2} \mu}{\sigma^{2} \phi^{2} \Lambda^{2}}\right)}\right), \\
K_{e}(\omega)=\frac{\gamma P_{0}}{\gamma-(\gamma-1)(1+H \sqrt{1+j \omega / H} / j 2 \omega)},
\end{gathered}
$$

where $\rho_{0}$ is the air density, $\gamma$ is the fluid specific heat ratio, $P_{0}$ is the fluid ambient pressure and $H=16 \mu /\left(B^{2} \Lambda^{\prime 2} \rho_{0}\right)$. In the last expression, $B^{2}$ is the Prandtl number. The wave number and characteristic impedance of the material as an equivalent fluid are then easily obtained as

$$
\mathrm{k}_{\mathrm{e}}(\omega)=\omega \sqrt{\widetilde{\rho}_{\mathrm{e}}(\omega) / K_{e}(\omega)} \quad \text { and } \quad \mathrm{Z}_{\mathrm{e}}(\omega)=\sqrt{\tilde{\rho}_{\mathrm{e}}(\omega) K_{e}(\omega)} .
$$


In the equivalent fluid modelling, only the absorption curve is visualized and the effect of the resonant structure is not accounted for. Since in the numerical model the resonant structure is assumed rigid, two variations for each type (constant and non-constant) are seen:

1. A so-called high frequent design for which no stop band is expected in the region of increased acoustic absorption.

2. A so-called low frequent design for which a stop band is expected in the region of absorption peak.

For the constant thickness case, the mass of air around the resonant structure mass and legs moves at the same rate, similar to a slit shape microperforated panel in which case the length correction yields $\varepsilon=0.48 \sqrt{\pi r_{h}{ }^{2}}(1-1.25 \sqrt{\phi})$, as indicated above. Figure 9 shows the real and imaginary parts of the impedance of a constant thickness metamaterial. The equivalent fluid model with correction lengths for rounded edges is compared to the model of slit-shaped perforations as described by Randeberg in [10]. For the frequency range of interest, both models are in good agreement confirming that the constant thickness resonant structure behaves purely as a microslit panel as long as no resonance effect appears [20].

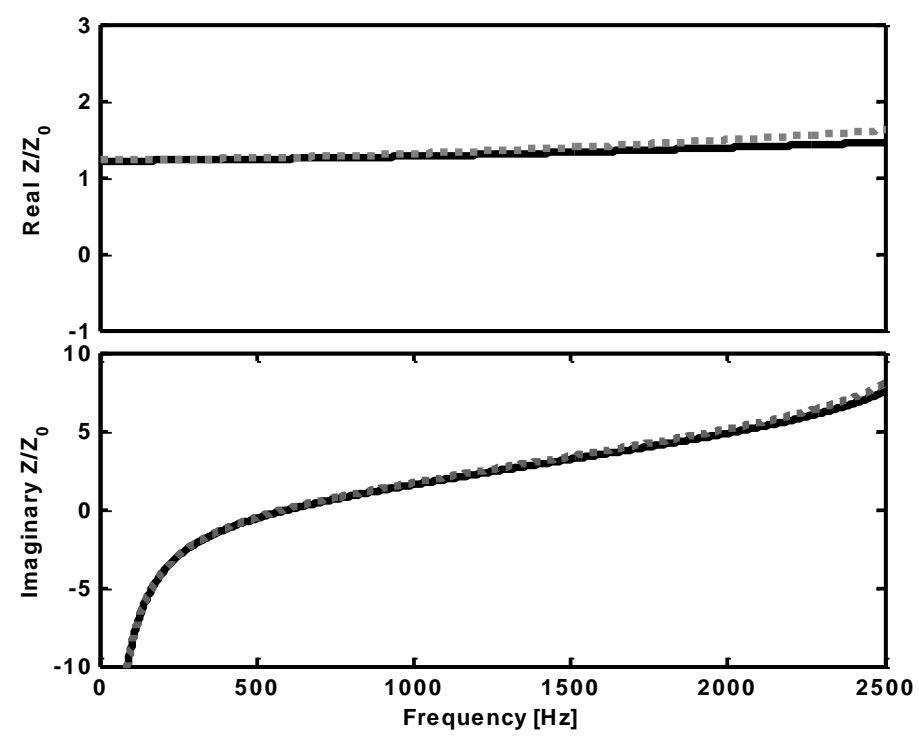

Figure 9: Real and imaginary part of the impedance of a constant thickness resonant structure with $T_{\mathrm{r}}=4 \mathrm{~mm}, d=0.2 \mathrm{~mm}$ and backing cavity $D=6 \mathrm{~cm}$. Randeberg model (in straight line) compared with equivalent fluid model (in dotted line) with $\varepsilon=0.48 \sqrt{\pi r_{h}^{2}}(1-1.25 \sqrt{\phi})$.

There are two cases for which this correction length needs to be adapted. Firstly, in the case of a non-constant thickness resonant structure, since the volume of air radiated by the legs and the mass have different depths, two volumes of air are joined together in the intersection between slits $\mathrm{A}$ and $\mathrm{B}$ of Figure 7. This effect is analogue to the case of two apertures combined into one resulting in an over perforated unit cell where the synergetic effect of the movement of both sections is expected to modify the correction length. As observed by Ingard [20], a correction length higher by a factor of $\sqrt{2}$ can account for this effect. Therefore, $\varepsilon_{m}=\sqrt{2} \varepsilon$ is used for this case. Secondly, a similar reasoning can be followed when the absorption due to the microslits and the backing cavity is in the frequency zone in which the resonant structures are moving relatively to the host structure. From Figure 3 it is clear that the air in the slits surrounding the resonant structure mass will move at a different rate than the mass of air at the slits surrounding the 
resonant structure legs. Therefore, $\varepsilon_{m}=\sqrt{2} \varepsilon$ is used as well for the constant thickness lay-out if movement of the resonant structure is expected. The examples for which this is the case will be clearly indicated in Sections 4.2 and 4.3.

The slit width and backing cavity depth are tuned with respect to frequency and strength of the absorption peak. This allows matching the region of good transmission loss of the metamaterials, with an increased absorption peak as well. As a result, a highly absorptive metamaterial tuned for a resonance frequency in the low to mid frequency range and in one to two octave bands is created. To validate this approach, the absorption coefficient is measured in an adapted impedance tube, as will be shown in Section 4.

\subsection{Acoustic field description by matrix representations}

A straightforward way of obtaining the normal incidence surface impedance of a material is by using the transfer matrix approach (TMM). By making appropriate assumptions, an acoustic element can be represented by a [2x2] matrix that relates its upstream and downstream pressures and velocities [15]. Many examples and applications of the TMM found in literature describe systems which have layers in series configurations. This is the case of the constant thickness resonant structure when it is placed in front of a rigid wall. However, the TMM has also been used for parallel assemblies or patchworks allowing expanding its use towards composite materials of parallel constructions [15, 21]. As exposed in Section 2.2, since the mass of the resonant structure is thicker than the legs, a non-constant thickness resonant structure consists of two clearly identifiable sections (Figure 6, left). The combination between the legs and an air cavity of thickness $D_{a}=T_{r}-T_{s}$ is interpreted as two layers in series that are at the same time in parallel with the resonator mass. In this framework and making the assumption that all components i.e., the mass, the legs, and the air cavity $D_{a}$, belong to an equivalent fluid, it is simple to see that the transfer matrices in parallel and in series are the appropriate methods to predict the acoustic absorption of the structure. Therefore the series and parallel transfer matrix are explained. In a series assembly, each layer forms a one-dimensional acoustic element whose transfer matrix is written as [22]

$$
\left[\begin{array}{l}
\mathrm{p}_{\mathrm{n}} \\
\mathrm{u}_{\mathrm{n}}
\end{array}\right]=\left[\begin{array}{ll}
t_{\mathrm{e}, 11} & t_{e, 12} \\
t_{e, 21} & t_{e, 22}
\end{array}\right]\left[\begin{array}{l}
\mathrm{p}_{\mathrm{n}+1} \\
\mathrm{u}_{\mathrm{n}+1}
\end{array}\right]
$$

where $p_{n}, u_{n}$ and $p_{n+1}, u_{n+1}$ are the pressure and particle velocity upstream and downstream of the acoustic element and $\mathrm{T}_{\mathrm{e} 11}, \mathrm{~T}_{\mathrm{e} 12}, \mathrm{~T}_{\mathrm{e} 21}, \mathrm{~T}_{\mathrm{e} 22}$ are the transfer matrix elements. For instance, this element can be a porous foam, a perforated plate, an equivalent fluid, or a gas or fluid. Since the structure under study can be considered an equivalent fluid, the transfer matrix is given by

$$
\left[\begin{array}{l}
\mathrm{p}_{\mathrm{n}} \\
\mathrm{u}_{\mathrm{n}}
\end{array}\right]=\left[T_{e}\right]\left[\begin{array}{l}
\mathrm{p}_{\mathrm{n}+1} \\
\mathrm{u}_{\mathrm{n}+1}
\end{array}\right]=\left[\begin{array}{cc}
\cos k_{\mathrm{e}} t_{\mathrm{e}} & \frac{\mathrm{j} Z_{\mathrm{e}}}{\phi_{\mathrm{e}}} \sin k_{\mathrm{e}} t_{\mathrm{e}} \\
\frac{\mathrm{j} \phi_{\mathrm{e}}}{Z_{\mathrm{e}}} \sin k_{\mathrm{e}} t_{\mathrm{e}} & \cos k_{\mathrm{e}} t_{\mathrm{e}}
\end{array}\right]\left[\begin{array}{l}
\mathrm{p}_{\mathrm{n}+1} \\
\mathrm{u}_{\mathrm{n}+1}
\end{array}\right],
$$

where $j^{2}=-1, k_{e}, t_{e}, \phi_{e}$ and $Z_{e}$ are the wave number, the thickness, the porosity and the impedance of the fluid, respectively. The global transfer matrix $T_{\mathrm{G}}$ of an acoustic assembly consisting of an equivalent fluid in series with a layer of air $T_{\text {air }}$ can be obtained by the multiplication of the individual matrices of the elements, i.e. $T_{\mathrm{G}}=T_{\mathrm{e}} T_{\mathrm{air}}$. The normal incidence surface impedance is obtained as

$$
\mathrm{Z}_{\mathrm{i}}=\frac{t_{G 1,1}}{t_{G 2,1}}
$$


As explained by Verdière et. al. for a parallel assembly, instead of working with the impedance matrix, the admittance matrix $\mathrm{Y}_{\mathrm{e}}$ is used. In this way, Eq. (12) can be rewritten as:

$$
\left[\begin{array}{c}
\mathrm{u}_{\mathrm{n}} \\
\mathrm{u}_{\mathrm{n}+1}
\end{array}\right]=\left[\begin{array}{cc}
\mathrm{y}_{\mathrm{e}, 11} & \mathrm{y}_{\mathrm{e}, 12} \\
\mathrm{y}_{\mathrm{e}, 21} & \mathrm{y}_{\mathrm{e}, 22}
\end{array}\right]\left[\begin{array}{c}
\mathrm{p}_{\mathrm{n}} \\
\mathrm{p}_{\mathrm{n}+1}
\end{array}\right]
$$

and

$$
\left[\begin{array}{c}
u_{n} \\
u_{n+1}
\end{array}\right]=\left[Y_{e}\right]\left[\begin{array}{c}
p_{n} \\
p_{n+1}
\end{array}\right]=\frac{1}{t_{e, 12}}\left[\begin{array}{cc}
t_{e, 22} & t_{e_{, 21}} t_{e, 12}-t_{e, 22} t_{e, 11} \\
1 & -t_{e, 11}
\end{array}\right]\left[\begin{array}{c}
p_{n} \\
p_{n+1}
\end{array}\right]
$$

The continuity of pressure at each element is imposed by the assumption of plane wave propagation. The admittance system of a number of elements in parallel is retrieved by combining the expressions for the continuity of the pressure and the normal component of the particle velocity with Eq. (15) yielding

$$
\left[\begin{array}{c}
\mathrm{u}_{\mathrm{n}} \\
\mathrm{u}_{\mathrm{n}+1}
\end{array}\right]=\left(\sum r_{e}\left[\mathrm{Y}_{e}\right]\right)\left[\begin{array}{c}
\mathrm{p}_{\mathrm{n}} \\
\mathrm{p}_{\mathrm{n}+1}
\end{array}\right]
$$

where $r_{e}$ is the quotient between the cross-sectional surface area $S_{e}$ of the element and the total surface area $S_{\text {total }}$ under study. In order to have an expression in terms of a transfer matrix, Eq. (17) is rearranged to express the sound pressure and particle velocity at the upstream and downstream sides of the element. The transfer matrix $T_{\mathrm{G}}$ of all the elements in parallel yields

$\left[\begin{array}{c}\mathrm{p}_{\mathrm{n}} \\ \mathrm{u}_{\mathrm{n}}\end{array}\right]=\mathrm{T}_{G}\left[\begin{array}{c}\mathrm{p}_{\mathrm{n}+1} \\ \mathrm{u}_{\mathrm{n}+1}\end{array}\right]=\left[-\frac{1}{\sum \mathrm{r}_{\mathrm{e}} \mathrm{y}_{\mathrm{e}, 21}}\left(\begin{array}{cc}\sum \mathrm{r}_{\mathrm{e}} \mathrm{y}_{\mathrm{e}, 22} & -1 \\ \sum \mathrm{r}_{\mathrm{e}} \mathrm{y}_{\mathrm{e}, 22} \sum \mathrm{r}_{\mathrm{e}} \mathrm{y}_{\mathrm{e}, 11}-\sum \mathrm{r}_{\mathrm{e}} \mathrm{y}_{\mathrm{e}, 12} \sum \mathrm{r}_{\mathrm{e}} \mathrm{y}_{\mathrm{e}, 21} & -\sum \mathrm{r}_{\mathrm{e}} \mathrm{y}_{\mathrm{e}, 11}\end{array}\right)\right]\left[\begin{array}{l}\mathrm{p}_{\mathrm{n}+1} \\ \mathrm{u}_{\mathrm{n}+1}\end{array}\right]$.

The absorption coefficient at normal incidence is calculated as

$$
\alpha=1-\left|\frac{T_{G 11}-Z_{0} T_{G 21}}{T_{G 11}+Z_{0} T_{G 21}}\right|^{2},
$$

with $Z_{0}=\rho_{0} c_{0}$, were $c_{0}$ is the speed of sound in air. The use of the parallel approach is valid as long as the following assumptions hold: upstream and downstream plane wave propagation, locally reacting materials, no pressure diffusion between adjacent parallel elements, wavelengths larger than the unit cell and representation of each element by a $2 \times 2$ matrix. From Figure 6, it is observed that no impervious wall separates the parallel constituents of the structure and as such, the non-exchange assumption is no longer valid. However, pressure uniformity is assured by homogeneously spreading the unit cells onto the assembly. Moreover, for the thicknesses studied, $k_{\mathrm{e}} t_{\mathrm{e}} \ll 1$ and the equivalent fluids of the parallel transfer matrix can be homogenized by the mixing rules method as described in [23].

\section{$4 \quad$ Numerical and experimental results}

This section starts with an overview of the test set-up and a discussion on the production of the test samples. Next the numerical and experimental results for the different microslit metamaterial samples are compared and discussed.

\subsection{The test set-up and the test samples}

An adapted aeroacoustic test-rig shown in Figure 10 is used for the impedance characterization of the samples. The tube section consists of a straight duct of length $2.1 \mathrm{~m}$ with a square cross section of $0.04 \times 0.04 \mathrm{~m}$. Four pressure transducers PCB 378C10 are flush-mounted in the upper 
side of the duct and located at distances $0.04,0.12,0.25$ and $0.46 \mathrm{~m}$ from the sample. At the opposite side of the sample, a loudspeaker able to generate broadband plane waves is located. The loudspeaker cavity is $0.22 \mathrm{~m}$ long and the output has a circular geometry. To adapt the geometries of the loudspeaker cavity to the square tube, a $0.21 \mathrm{~m}$ duct with one circular and one square termination is placed between both sections. The air properties during the measurements are: $T=19.5^{\circ} \mathrm{C}, \rho_{0}=1.2062 \mathrm{~kg} / \mathrm{m}^{3}, c_{0}=343.26 \mathrm{~m} / \mathrm{s}, \quad B^{2}=0.7087, \gamma=1.399, \mu=$ $1.81 \times 10^{-5} \mathrm{Pas}$ and $P_{0}=101600 \mathrm{~Pa}$. The low $f_{l}$ and high $f_{u}$ cut-on frequencies for a valid measurement range are found to be $f_{l}=c_{0} / 20\left|z_{0}-z_{1}\right|=431 \mathrm{~Hz}$ and $f_{u}=0.45 c_{0} / \mid z_{0}-$ $z_{1} \mid=3879 \mathrm{~Hz}$, where $z_{0}$ corresponds to the location of the sample and $z_{1}$ is the distance from the sample to the nearest microphone. The pressure fluctuations are measured with LMS TestLab $13 \mathrm{~A}$ at the microphone locations and the impedance is retrieved by using an iterative procedure as described in [24]. To allow a maximum absorption to occur in the microslit metamaterials and in order to generate enough viscous losses as the air traverses the structure, the slit diameter is designed to be $0.4 \mathrm{~mm}$ and the sample is placed in front of a cavity filled with a mass of air and backed by a rigid wall. Different backing cavities are used to observe the effect of the resonant structures in the absorption. For each test, the backing cavity is made by addition of tube sections with different thicknesses until the preferred dimension is achieved. The microslit metamaterials are clamped between the tube and the additional tube sections with four screws, downstream the microphones.

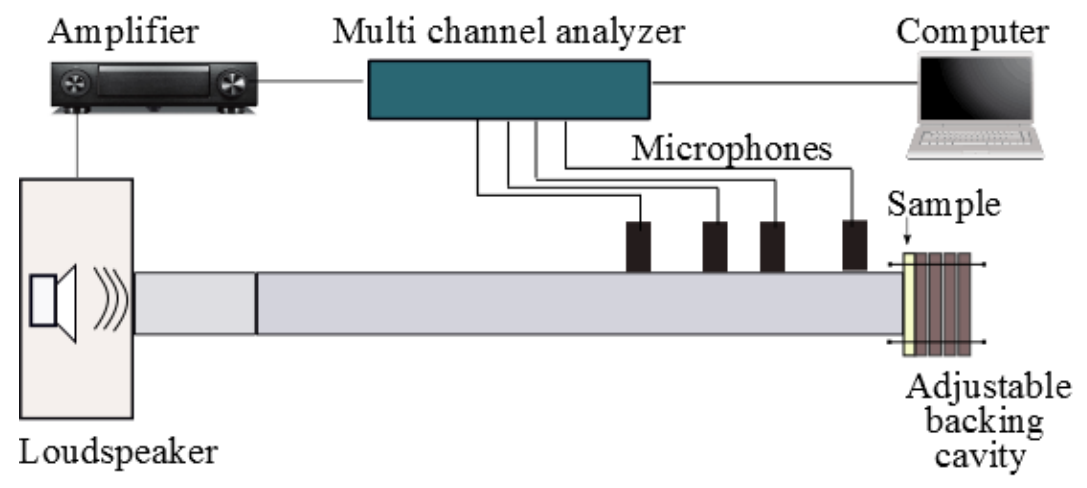

Figure 10: Schematic representation of the adapted aeroacoustic test-rig.

The samples are 3D printed and laser cutting is used to completion since some of the slits were not fully open (Figure 11). As a result, the slit diameter in each sample has some spread. To obtain a standard diameter to be used in the numerical models, all slits are measured using a microscope and the average value per sample is found. Table 4 shows the average diameter obtained and the porosities calculated for each sample.

\begin{tabular}{|c|c|c|c|c|}
\cline { 2 - 5 } \multicolumn{1}{c|}{} & \multicolumn{2}{c|}{ Constant thickness } & \multicolumn{2}{c|}{ Non-constant thickness } \\
\cline { 2 - 5 } \multicolumn{1}{c|}{} & Low freq. & High freq. & Low freq. & High freq. \\
\hline$d$ & $0.4 \mathrm{~mm}$ & $0.313 \mathrm{~mm}$ & $0.49 \mathrm{~mm}$ & $0.43 \mathrm{~mm}$ \\
$\phi_{c}$ & $8.70 \%$ & $6.81 \%$ & $10.66 \%$ & $9.63 \%$ \\
\hline
\end{tabular}

Table 4: Average diameter and porosities of the microslit metamaterials. 
Given the tube section used and since each elementary cell is $11.5 \mathrm{~mm}$ long, 9 resonant structures have been distributed in a square lattice as shown in Figure 11.
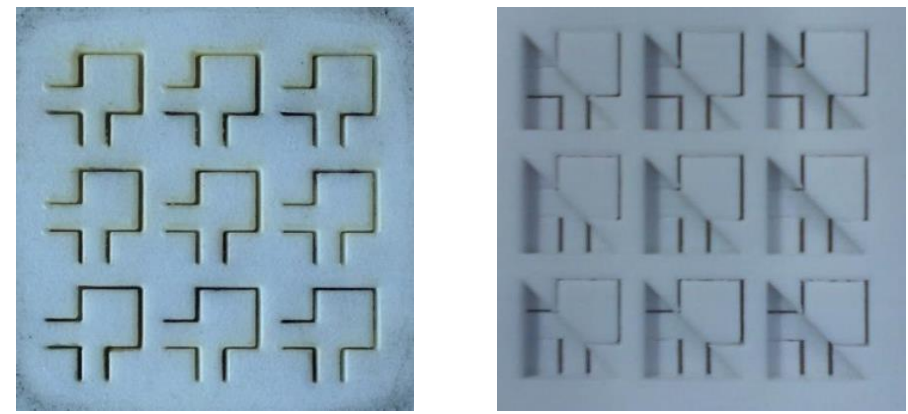

Figure 11: Front view of a constant thickness (left) and a non-constant thickness resonant structure (right). The left side shows a sample after laser cutting to guarantee that all slits are open, the right side shows a sample as 3D-printed before the laser cutting action.

In view of interpreting the test results, dynamic structural models of the test samples are analysed. The FE model is built as a repetition of the unit cell models, described in Section 2. Since perfect clamping cannot be accomplished, it is challenging to mimic the boundary condition of the test set-up. Consequently, two different boundary conditions are used each time: a clamping condition by setting the degrees of freedom on the boundary of the models to zero and a support condition by setting the degrees of freedom on the bottom edges of the side to zero. In the case of shell elements, a supported edge (no displacements) is compared to a fully clamped edge (no displacements and rotations). The real mounting conditions are somewhere in between these settings.

A previous paper of the authors discusses the effect of a stop band on the structural modes in case of supported boundaries [25]: it is expected that for the frequency zone below the stop band 9 modes will be found where the 9 resonant structures move in phase with the displacement of the sample. Within the stop band, no modes are to be found, while right after the stop band, modes will be found with the resonant structures moving out of phase with respect to the host structure. For clamped boundaries similar conclusions are expected [26], however, the clamping will influence some of the resonance frequencies of the resonant structures since some boundaries of unit cells become stiffer. From Figure 11 it can be seen that five of the nine resonant structures have at least one connection leg close to the boundary. The stiffer boundary condition will increase the stiffness of this connection leg and thus lead to a shift in resonance frequency of the resonant structure; this will result to a shift in stop band region or to modes appearing in the stop band region predicted by the unit cell model. This will be most pronounced in the case for the more flexible structures such as the constant thickness resonant structure lay-out.

\subsection{Case 1: Constant thickness resonant structure lay-out}

With the purpose of controlling noise in the low to mid-frequencies and due to the test-rig restrictions, the targeted range of absorption was set between 500 and $3500 \mathrm{~Hz}$. A first sample with a higher frequency stop band was designed $\left(T_{r}=4 \mathrm{~mm}\right)$ such that the stop band is well outside of the intended area for good acoustic absorption. The first and second structural mode of the sample are predicted at $3112 \mathrm{~Hz}$ and $4436 \mathrm{~Hz}$ for clamped boundaries and $2257 \mathrm{~Hz}$ and 3440 $\mathrm{Hz}$ for supported boundaries, which is high enough in frequency to not interfere with the acoustic absorption due to the microslits. The second sample, with a lower frequent stop band is made with an overall thickness of $1 \mathrm{~mm}$. According to the Finite Element model this structure provides 
a stop band between 1526-1968 Hz, (Table 3). Measuring both samples in the same frequency range will allow investigating the effect of the stop band on the frequency range of absorption.

Both the low and high frequent designs are measured with two different backing cavities of $D_{1}=5.2 \mathrm{~cm}$ and $D_{2}=3 \mathrm{~cm}$, respectively. For each measurement, the mean absorption is calculated between the two frequencies at which the absorption is $50 \%$ of the maximum peak. In this case this yields $61 \%$ for $D_{1}$ and $64 \%$ for $D_{2}$. The results are compared with the slitted panel model of Randeberg and the equivalent fluid modelling using the transfer matrix in series.

Figure 12 shows the measured absorption curve of the high frequent design with the two backing cavities compared with theory. In general, an excellent agreement is seen between theory and measurements. Furthermore, by comparing the experimental results with the slitted panel theory, it is confirmed that for the targeted frequency range, the constant thickness resonant structure behaves purely as a microslit panel.

A peak in absorption appears around $2780 \mathrm{~Hz}$ for $D_{1}$ and at $2774 \mathrm{~Hz}$ for $D_{2}$. These peaks correspond with the frequency zone for which the first structural mode of the sample is expected. Given the method used to clamp the samples, a perfect repeatable clamping cannot be achieved. As the mounting condition has an impact on the first structural mode of the sample, a slight shift in the location of the peak is seen for the two different backing cavities.

By decreasing the back cavity depth, the peak of absorption due to the microslits can be shifted to an increased frequency to reach the zone were the first structural mode is found. As means of demonstration, the high frequency constant thickness sample was measured with a smaller backing cavity in order to place the absorption peak to the higher frequencies. Figure 13 shows that the band of absorption is widened by the effect of the two absorption peaks when they are located very close to each other. The mean absorption obtained for a cavity $D_{1}=0.6 \mathrm{~cm}$ is $69 \%$ which is higher compared with the two previous cavities. It is observed that the slitted panel model of Randeberg can no longer account for this combined effect and the equivalent fluid model presents a good agreement when the correction length is taken as $\varepsilon_{m}=\sqrt{2} \varepsilon$, as explained in Section 3.1.

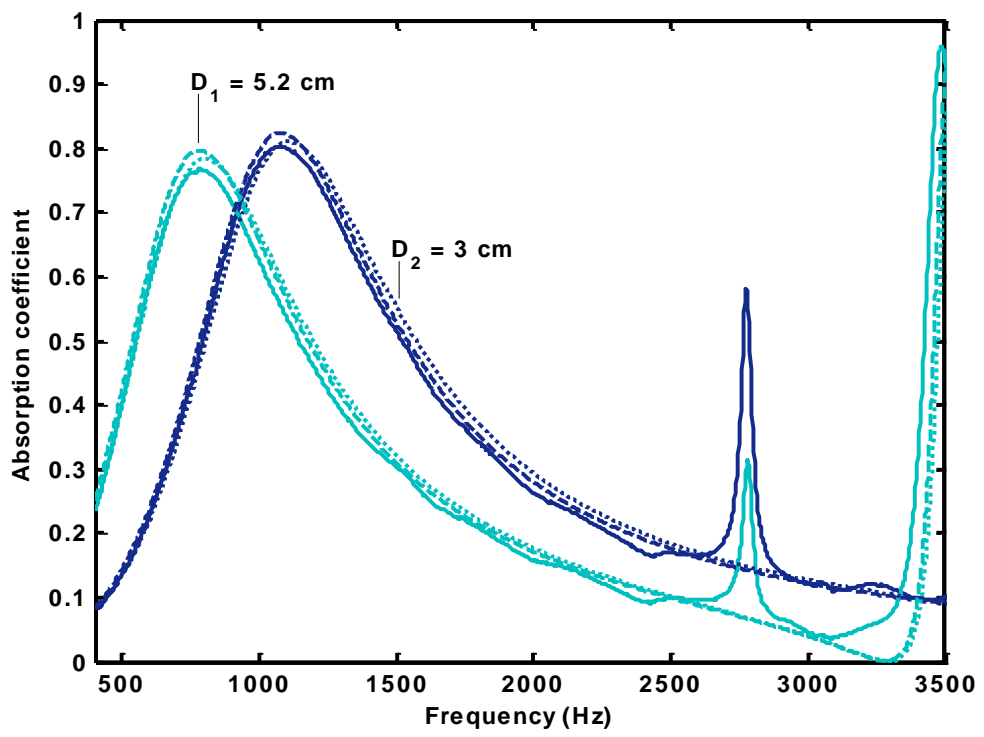

Figure 12: Absorption coefficient at normal incidence of the high frequent constant thickness resonant structure. - Solid line measured, … Dotted line Randeberg model, --- Dashed line equivalent fluid model. 


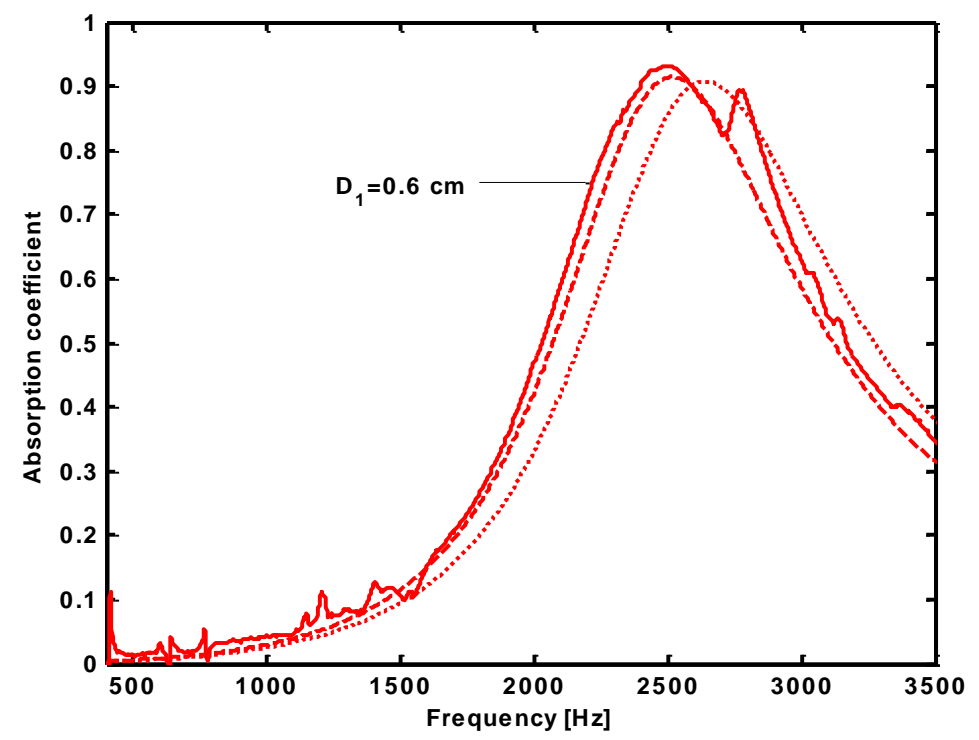

Figure 13: Absorption coefficient at normal incidence of the high frequent constant thickness resonant structure in the structural resonance range. - Solid line measured, ${ }^{\cdots \cdot .}$ Dotted line Randeberg model, --- Dashed line equivalent fluid model with $\varepsilon_{m}=\sqrt{2 \varepsilon}$.

The first eleven structural modes of the low frequent constant thickness sample are shown in Table 5. The expected stop band is located between $1526 \mathrm{~Hz}$ and $1968 \mathrm{~Hz}$. The peak of absorption is tuned for this frequency range by setting the air backing cavity equal to $5.2 \mathrm{~cm}$. To shift the absorption peak to higher frequencies, the sample is measured once more with a backing cavity of $3 \mathrm{~cm}$.

\begin{tabular}{c|ccccccccccc} 
Mode & 1 & 2 & 3 & 4 & 5 & 6 & 7 & 8 & 9 & 10 & 11 \\
\hline Freq. sup. (Hz) & 502 & 1063 & 1117 & 1359 & 1406 & 1418 & 1496 & 1504 & 1532 & 2067 & 2225 \\
\hline Freq. clamp. (Hz) & 946 & 1488 & 1567 & 1660 & 1694 & 1722 & 1760 & 1776 & 1940 & 2177 & 2598
\end{tabular}

Table 5: First 11 predicted structural modes of the low frequent constant thickness sample.

By inspecting the measured results shown in Figure 14, it is observed that the first nine modes appear as small peaks on the absorption curve followed by a region were no pronounced peaks are observed and thus confirming the location of the stop band. Again, the equivalent fluid model of the sample in series with an air cavity presents excellent agreement with measured results. 


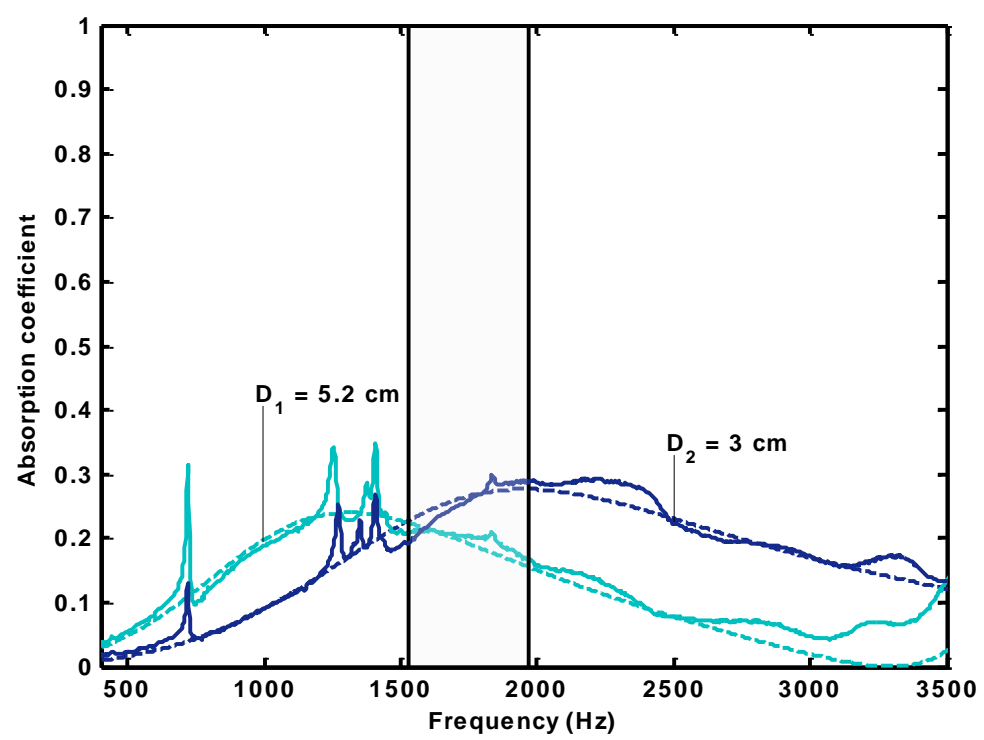

Figure 14: Absorption coefficient at normal incidence of the low frequent constant thickness resonant structure. - Solid line measured, --- Dashed line equivalent fluid model, stopband region shaded.

\subsection{Case 2: Non-constant thickness resonant structure lay-out}

The goal of the non-constant thickness lay-outs is to provide more freedom in tuning the resonance frequency of the resonant structure and to bring it to lower frequencies. Again a low frequent and a high frequent design are studied. The low frequent design has a stop band predicted to be from $1011 \mathrm{~Hz}$ to $1180 \mathrm{~Hz}$. The high frequent resonant design has an increased leg thickness, such that the resonance frequency is again shifted higher in frequency. With an air cavity equal to $5.2 \mathrm{~cm}$, both designs should provide absorption near the frequency band of 968 $\mathrm{Hz}$, such that the high frequent design allows validating the model, and the low frequent design can be used to investigate a possible effect of the resonant structure movement in the zone of higher absorption. The first 11 modes of the high frequency design are shown in Table 6.

\begin{tabular}{c|ccccccccccc} 
Mode & 1 & 2 & 3 & 4 & 5 & 6 & 7 & 8 & 9 & 10 & 11 \\
\hline Freq. sup. (Hz) & 1817 & 2175 & 2193 & 2238 & 2265 & 2268 & 2334 & 2351 & 2369 & 3373 & 4684 \\
\hline Freq. clamp. (Hz) & 2173 & 2367 & 2387 & 2415 & 2484 & 2492 & 2497 & 2499 & 2583.7 & 4190 & 5337
\end{tabular}

Table 6: First 11 predicted structural modes of the high frequency non-constant thickness sample

As observed by the experimental results of Figure 15, the absorption peak due to the microslits appears in a frequency region below the structural modes, which start acting as small spikes after $1817 \mathrm{~Hz}$. The stop band region is expected to be from $2374 \mathrm{~Hz}$ up to $2841 \mathrm{~Hz}$ which manifests itself in Figure 15. In this region no abrupt peaks show on top of the predicted absorption curve. 


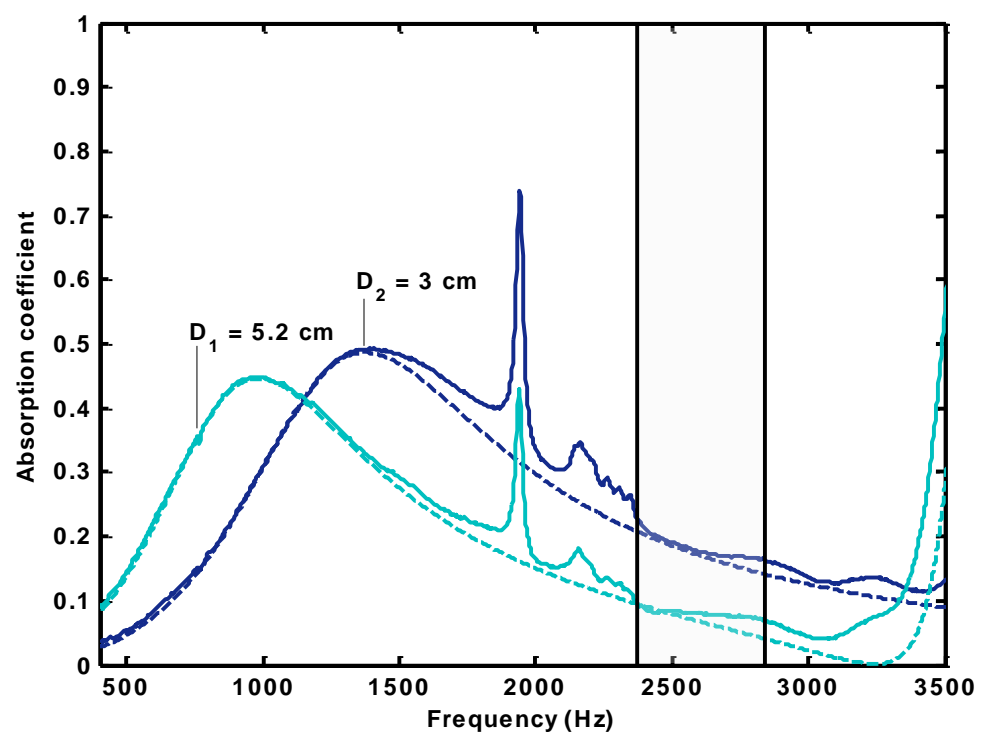

Figure 15: Absorption coefficient at normal incidence of the high frequent non-constant thickness resonant structure. - Solid line measured, --- Dashed line equivalent fluid model, stopband region shaded.

The low frequency case is shown in Figure 16. In this example, the structural modes of the sample appear in the low frequency region and as observed before in the constant thickness resonant case, the peaks of each mode are smaller as compared to the peaks presented by the high frequency case. Table 7 shows the modes of the structure, the predicted structural modes of the sample appear to be almost $200 \mathrm{~Hz}$ above the measured peaks in absorption. Again, this behaviour might be attributed to the clamping of the sample.

\begin{tabular}{c|cccccccccccc} 
Mode & 1 & 2 & 3 & 4 & 5 & 6 & 7 & 8 & 9 & 10 & 11 \\
\hline Freq. sup. (Hz) & 970 & 977 & 989 & 990 & 992 & 998 & 1008 & 1010 & 1011 & 2552 & 3118 \\
\hline Freq. clamp. (Hz) & 1001 & 1010 & 1012 & 1014 & 1019 & 1019 & 1020 & 1020 & 1028 & 3158 & 3199
\end{tabular}

Table 7: First 11 predicted structural modes of the low frequent non-constant thickness sample

Comparing the absorption regions of the two non-constant thickness cases, it is observed that the high frequency case provides a greater absorption peak as compared to the low frequency case. This effect is not related to the modes of the test sample but to the legs thickness. Viewing the legs part as a microperforated layer, it is expected that a microperforated panel of $2 \mathrm{~mm}$ thickness provides a higher absorption than a $1 \mathrm{~mm}$ thick panel. 


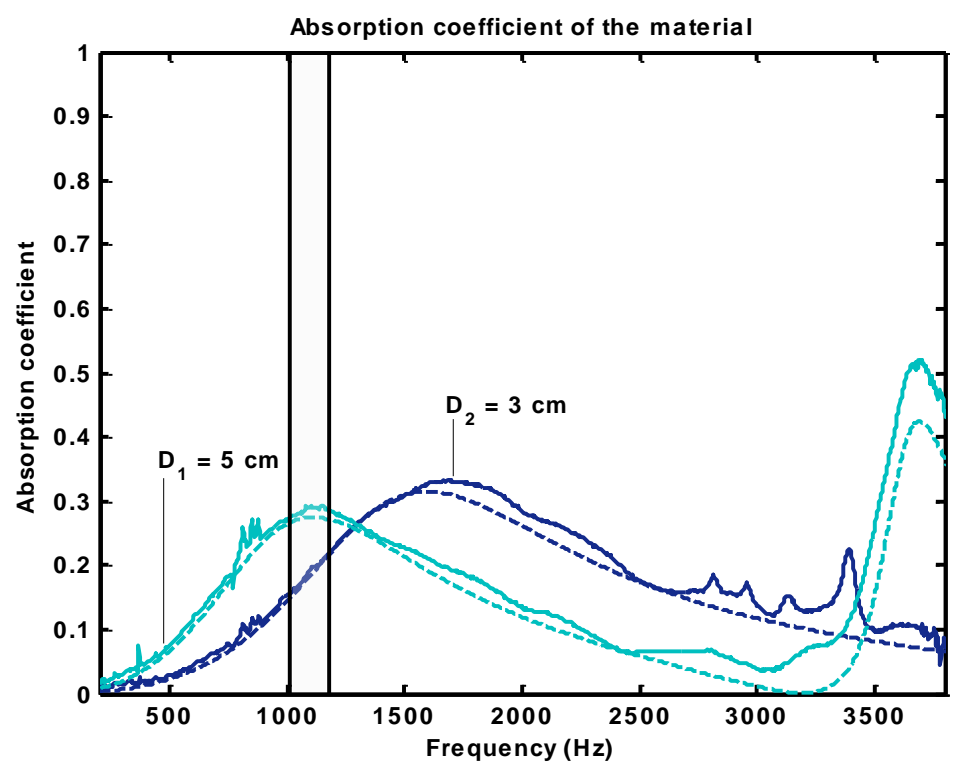

Figure 16: Absorption coefficient at normal incidence of the low frequent non-constant thickness resonant structure. - Solid line measured, --- Dashed line equivalent fluid model, stopband region shaded.

\section{Conclusions}

In this paper, the acoustic characteristics of a resonant based metamaterial have been modified in order to provide acoustic absorption. It is shown that by decreasing the air gap of the elementary cell of a metamaterial and leaving micro-diameter air slits between the host structure and the mass and legs of the resonant structure, acoustic absorption is achieved by viscous losses. Constant and non-constant thicknesses resonant structures designs are proposed for which low and high frequency cases are studied. It is seen that a high frequency constant thickness resonator with slits of micrometric diameter behaves purely as a slit panel and its absorption can be calculated with traditional models for micro-slit panels. On the contrary, the equivalent fluid model is found to be more appropriate to predict the acoustic impedance of the low frequency constant case as well as the non-constant thickness examples. Furthermore, it is demonstrated that the unit cell of the non-constant thickness microslit metamaterial can be divided in parallel layers whose impedance can be obtained by using a transfer matrix TMM approach in series and parallel. FEM models allow predicting the structural response of resonating structures and the stop band regions, complementing the use of the equivalent fluid modelling which accounts for the visco-thermal effects. Thus, the absorption and structural effects are studied separately but observed in a combined way in the measurements. The experimental results reveal the presence of the structural modes of each test sample as pronounced peaks on the absorption curve. It is observed that the structural modes of the high frequency cases lead to higher absorption peaks than the modes in the low frequency cases. This is attributed to the thickness of the legs as the high frequent resonant structures are thicker and provide a higher amount of energy loss. Nevertheless, in all cases the structural modes and the stop band region are clearly present. The proposed design can clearly be improved, starting from the optimization of the shapes and geometries to the materials used, the methods of production and its costs. Further research is needed to study the acoustic transmission of the structural metamaterial with and without the microslits and to develop a model that combines the acoustic dissipation with the structural 
interaction. Yet, the microslit metamaterials presented in this study already set a step further towards the understanding, improvement and development of new lightweight and efficient materials with excellent acoustic and mechanical properties.

\section{Acknowledgments}

The authors would like to acknowledge the European Commission through the FP7-PEOPLEITN-2011 FlowAirS project (http://www.flowairs.eu), grant agreement 289352, from which the first author, Dr. Heidi Ruiz, held a grant. Furthermore, the authors would like to acknowledge the European Commission for their support through the ENLIGHT project (http://www.projectenlight.eu/) and the KU Leuven Research Fund for their support through an IOF Leverage project. Elke Deckers is a postdoctoral fellow of the Fund for Scientific Research Flanders (F.W.O. Vlaanderen).

\section{References}

[1] C. C. Claeys, B. Pluymers, P. Sas, and W. Desmet, "On the potential of tuned resonators to obtain low-frequency vibration stop bands in periodic panels," J Sound Vib, vol. 332, pp. 1418-1436, 2013.

[2] C. Goffaux, J. S. Dehesa, A. L. Yeyati, P. Lambin, A. Khelif, J. Vasseur, and B. DjafariRouhani, "Evidence of fano-like interference phenomena in locally resonant materials," Physical Review Letters, vol. 88(22), p. 225502, 2002.

[3] F. Lemoult, N. Kaina, M. Fink, and G. Lerosey, "Wave propagation control at the deep subwavelength scale in metamaterials," Nature Physics, vol. 9, pp. 55-60, 2012.

[4] Z. Liu, X. Zhang, Y. mao YY Zhu, Z. Yang, C. T. Chan, and P. Sheng, "Locally resonant sonic metamaterials," Science, vol. 289(5285), pp. 1734-1736, 2000.

[5] J. Mei, G. Ma, M. Yang, Z. Yang, W. Wen, and P. Sheng, "Dark acoustic metamaterials as super absorbers for low-frequency sound," Nature communications, vol. 3, p. 756, 2012.

[6] D.-Y. Maa, "Potential of microperforated panel absorber," J Acoust Soc Am, vol. 104, pp. 2861-2866, 1998.

[7] J.-F. Allard and N. Atalla, Propagation of Sound in Porous Media. Modelling Sound Absorbing Materials. Wiley, Chichester, United Kingdom, 2009, chap. 5, .

[8] U. Ingard, "Perforated facing and sound absorption," J Acoust Soc Am, vol. 26(2), pp. 151-154, 1954.

[9] D.-Y. Maa, "Microperforated-panel wideband absorbers," Noise Control Eng J, vol. 29, pp. 77-84, 1987.

[10] R. Randeberg, "Perforated panel absorbers with viscous energy dissipation enhanced by orifice design," Ph.D.Thesis, Norwegian University of Science and Technology, Trondheim, 2000 .

[11] R. Langley., "A note on the force boundary conditions for two dimensional periodic structures with corner freedoms." J Sound Vib, vol. 167, p. 377-381, 1993.

[12] L. Brillouin, Wave propagation in periodic structures. McGraw-Hill Book Company, 2nd edition, 1946, New York, United States of America. 
[13] D. L. Johnson, J. Koplik, and R. Dashen, "Theory of dynamic permeability and tortuosity in fluid-saturated porous media," J. Fluid Mech., vol. 176, pp. 379-402, 1987.

[14] Y. Champoux and J. Allard, "Dynamic tortuosity and bulk modulus in air-saturated porous media," J. Appl. Phys., vol. 70, 1, pp. 975-1979, 1991.

[15] K. Verdière, R. Panneton, S. Elkoun, T. Dupont, and P. Leclaire, "Transfer matrix method applied to the parallel assembly of sound absorbing materials," J Acoust Soc Am, vol. 134(6), pp. 4648-4658, 2013.

[16] C. C. Claeys, E. Deckers, B. Pluymers, and W. Desmet, "A lightweight vibro-acoustic metamaterial demonstrator: Numerical and experimental investigation," Mech. Syst. Signal Process. (2015), http://dx.doi.org/10.1016/j.ymssp.2015.08.029.

[17] D. Maa, "Theory of microslit absorbers," Chinese J Acoust, vol. 20, pp. 1-10, 2001.

[18] N. Atalla and F. Sgard, "Modeling of perforated plates and screens using rigid frame porous models," J Sound Vib, vol. 303, pp. 195-208, 2007.

[19] H. Meng, J. Wen, H. Zhao, and X. Wen, "Optimization of locally resonant acoustic metamaterial on undewater sound absorption characteristics," J Sound Vib, vol. 331, pp. 44064416, 2012.

[20] U. Ingard, "On the theory and design on acoustic resonators," J Acoust Soc Am, vol. 25(6), pp. 1037-1061, 1953.

[21] K. Veridère, R. Panneton, S. Elkoun, T. Dupont, and P. Leclaire, "Prediction of acoustic properties of parallel assemblies by mean of transfer matrix method," in Proceedings of meetings on acoustics. ICA, vol. 19, no. 065011, 2013.

[22] D. H. Lee and P. Kwon, "Estimation of the absorption performance of multiple layer perforated panel systems by transfer matrix method.” J Sound Vib, vol. 278, pp. 847-860, 2004.

[23] K. Verdière, R. Panneton, S. Elkoun, T. Dupont and P. Leclaire, "Recent highlights on the parallel transfer matrix method (PTMM), in Symposium on the Acoustics of Poro-Elastic Materials, SAPEM. Stockholm, Sweden, 16-18 December 2014

[24] H. Denayer, W. de Roeck, and W. Desmet, "Iterative procedure for accurate plane wave decomposition in flow ducts," in International Conference on Noise and Vibration Engineering , ISMA. Leuven, Belgium, 15-17 September 2014.

[25] C. C. Claeys, P. Sas, and W. Desmet, "On the acoustic radiation efficiency of local resonance based stop band materials," J Sound Vib, vol. 333, 14, pp. 3203-3213, 2014.

[26] D. Mead and S. Parthan, "Free wave propagation in two-dimensional periodic plates," $J$ Sound Vib, vol. 64, p. 325-348, 1979. 